\title{
The Diagnostic Value of Multislice Computed Tomography (MSCT) and Semiotics of Lung Tissue Damage in the Early Stages of Clinical Manifestations of COVID-19
}

\author{
Petr M Kotlyarov ${ }^{1 *}$ and Dmitry G Soldatov ${ }^{2}$ \\ ${ }^{1}$ Federal State Budgetary Institution Russian Scientific Center of Roentgen radiology (RSCRR) of the Ministry of Healthcare of \\ the Russian Federation Address: 117997 Moscow, Russia
}

${ }^{2}$ N.I.Pirogov Russian National Research Medical University of the Ministry of Healthcare of the Russian Federation: 117997 Moscow, Russia

*Corresponding author: Petr M Kotlyarov, Federal State Budgetary Institution Russian Scientific Center of Roentgen radiology (RSCRR) of the Ministry of Healthcare of the Russian Federation Address: 117997 Moscow, Russia

\begin{tabular}{|c|c|}
\hline ARTICLE INFO & ABSTRACT \\
\hline Received: 㠅 July 17,2020 & Citation: Petr M Kotlyarov, Dmitry G Soldatov. The Diagnostic Value of Multislice \\
\hline Published: August 03, 2020 & $\begin{array}{l}\text { Computed Tomography (MSCT) and Semiotics of Lung Tissue Damage in the Early } \\
\text { Stages of Clinical Manifestations of COVID-19. Biomed J Sci \& Tech Res 29(2)-2020. } \\
\text { BJSTR. MS.ID.004777. }\end{array}$ \\
\hline
\end{tabular}

\section{Introduction}

Most publications cover MSCT-semiotics of lung tissue damage and assessment of the prevalence and development of the pathological process. However, the diagnostic value of MSCT in the early clinical manifestations of COVID-19, the detection and correct interpretation of MSCT images of lung injury during the first 3-5 days are still underestimated.

Aim of the Study: To describe the specific lung damage semiotics based on the MSCT-picture during the first 3-5 days of COVID-19 clinical symptoms manifestation.

Materials and Methods: Fifty six patients with a PCRconfirmed diagnosis of COVID-19 and suspected pneumonia were examined by MSCT at 3-5th days of disease clinical manifestation.

Results and Discussion: Five different early MSCT-types of viral pneumonia related to SARS-CoV-2 were identified at 3-5th days of clinical manifestations.

Pneumonia with focal lesions and limited spread were detected in $30.4 \%$ patients. One or several small foci (up to $30 \mathrm{~mm}$ ) of the Ground Glass Opacification (GGO) were detected in 1-3 segments of one or less often both lungs. The changes had indistinct external contours, were localized mainly in the peripheral parts of the lungs. In some foci, areas of high opacity were described, clearly distinguishable against the background of GGO. In $41.2 \%$ of these patients there was an increased lung markings of the type of reticular consolidation of the interstitium along the periphery of the lesions. In $11.8 \%$ patients with single foci of GGO clinical symptoms of COVID-19 were absent during the examination (the study was conducted at the request of the patient due to infection of relatives). In $17.6 \%$ patients, MSCT performed on the 3rd day after the onset of clinical symptoms of COVID-19 did not reveal the presence of typical lung injury. However, the second MSCT conducted on the 5 th day (2 days after the initial examination) due to a sharp deterioration in the patients' condition revealed signs of viral pneumonia related to COVID-19. Multifocal bilateral lesions type GGO up to 30-45 mm with damage from 3 to 5 lung segments, with indistinct, blurred outer edges, pleural reaction of the pericissuritis type, with interstitial thickening along the periphery of the lesions was found in $26.8 \%$ of patients. This group of patients differed from the previous one both in the polymorphism of the macrostructure of the foci and in the chaotic distribution of areas of the lung damage. The changes were observed in any part of the lungs, both near the pleura and in the middle, periapical zones of the lungs, and near of the mediastinal pleura. In $13.3 \%$ of patients, extensive bilateral 
changes were observed with several segments' lesions exclusively of the GGO type, against the background of which areas of swollen alveolar tissue were visualized.

Polysegmental bilateral lung lesion with predominantly peribronchial localization was observed in $23.2 \%$ patients. Several segments (sometimes, in different lobes) were involved in the process, the entire segment or its part was affected. The appearance against the background of GGO in the central zone of high opacity infiltration, air bronchogram sign, crazy paving pattern, thickening of the interalveolar, pulmonary interstitium of the honeycombing type, mediastinal lymphadenopathy, in some cases bounded pleural effusion, dilatation of the pulmonary veins of the affected area were described. Unlike the previous group, these patients showed predominantly peribronchial localization of pathological changes, dilatation, thickening of the bronchial septa, the appearance of a symptom of air bronchogram, and mediastinal lymphadenopathy. Some patients had local pleural effusion, crazy paving pattern - like areas of pulmonary interstitium strengthening, rough, cord-like, interstitium and interlobar pleura compaction. In contrast to the previous group, the size of the dense infiltrate in the central part of the focal changes in the GGO increased. Against this background, there was a "swelling" of some groups of alveoli, probably due to the defeat of the respiratory bronchioles.

Lobar infiltrative changes were detected in $12.5 \%$ of patients, pneumonia developed in 2-5 lobes, there was a combination of GGO foci, with dense infiltrates in the central zone, and Polysegmental infiltrates, with the appearance of air bronchogram sign , thickening of the bronchial septa, changes in the interstitium of the crazy paving type, reticular thickening of the interstitium, pleural reactions (in the form of pleural effusion, thickening of the pleura), mediastinal lymphadenopathy, dilatation of the peripheral pulmonary veins of the affected area. Atypical changes in the lung tissue were observed in $7.5 \%$ patients - an increase of lung marking due to diffuse compaction of the interstitium with damage to both lungs, thickening of the bronchial septa, dilatation of the pulmonary veins. The severity of GGO was moderate. The key point, in our opinion, of the emergence of the assumption of pneumonia caused by SARS-CoV-2 is the comparison of MSCT data with the clinical picture of the disease, monitoring of MSCT-data.

\section{Conclusion}

a) MSCT semiotics of early stages of pneumonia caused by SARS-CoV-2 has a specific macrostructure, which allows us to assume about the participation of this infection in the etiology of the disease.

b) MSCT is a necessary examination method for suspected COVID-19 pneumonia.

c) The different MSCT-pictures of the disease development are due to the different reactions of patients to SARS-CoV-2 infection.
ISSN: 2574-1241

DOI: $10.26717 /$ BJSTR.2020.29.004777

Petr M Kotlyarov. Biomed J Sci \& Tech Res

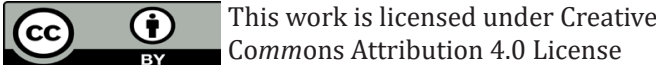

Submission Link: https://biomedres.us/submit-manuscript.php

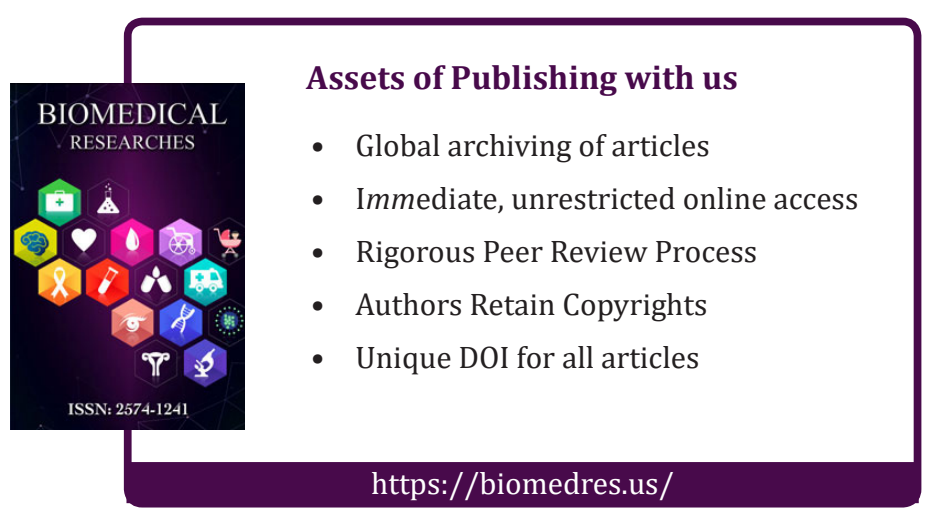

